\title{
Distúrbios osteomusculares relacionadas ao trabalho: Prevalência em trabalhadores
}

\section{de restaurantes}

\author{
Work-related musculoskeletal Disorders: Prevalence in restaurant workers \\ Trastornos musculoesqueléticas relacionadas con el trabajo: Prevalencia en trabajadores de \\ restaurantes
}

Recebido: 08/06/2021 | Revisado: 12/06/2021 | Aceito: 15/06/2021 | Publicado: 28/06/2021

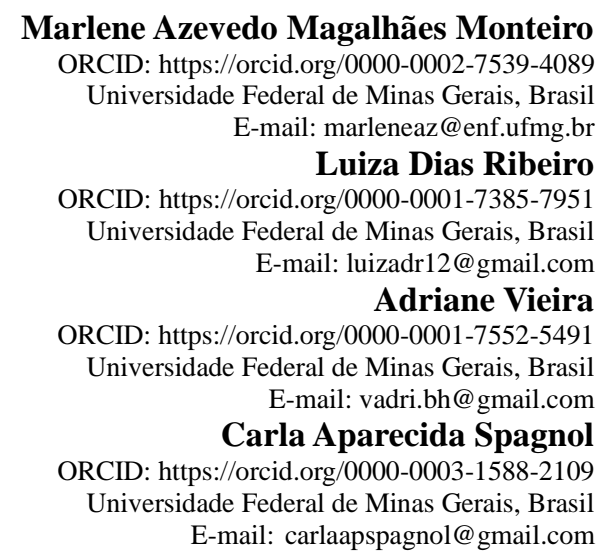

\section{Resumo}

O objetivo deste trabalho foi analisar a prevalência de Distúrbios Osteomusculares Relacionadas ao Trabalho (DORT) em trabalhadores de restaurantes universitários. Trata-se de um estudo transversal e descritivo com trabalhadores de quatro restaurantes universitários de Belo Horizonte/MG. A avaliação de DORT nos últimos doze meses foi investigada com aplicação do Questionário Nórdico de Sintomas Osteomusculares (QNSO), composto por questões fechadas e organizado por conjuntos temáticos que incluíram variáveis demográficas, sociofamiliares, autoavaliação de saúde, variáveis antropométricas, características ocupacionais e sintomas osteomusculares autorreferidos. Os dados foram tabulados e analisados pelo SPSS (p<0,05). Participaram da pesquisa 87 trabalhadores $(72,5 \%$ da amostra), com média da idade de $37 \pm 9,8$ anos, $81 \%$ do sexo feminino, tempo médio de trabalho de 9,3 \pm 7,5 anos, e jornada de trabalho de 40 horas semanais. Em relação à presença DORT nos últimos doze meses, prevaleceu a dor no ombro $(47,1 \%)$, inferior das costas $(40,2 \%)$, tornozelos/pés $(39,1 \%)$, e região temporal $(37,1 \%)$. Houve prevalência de sintomas osteomusculares associados a posturas, depressão, jornada de trabalho e realização de cursos de capacitação. Conclui-se que a atenção deve ser direcionada às posturas adotadas pelos trabalhadores de restaurantes na execução das tarefas a fim de reduzir a incidência de doenças ocupacionais.

Palavras-chave: Distúrbios Osteomusculares Relacionados ao Trabalho (DORT); Doenças ocupacionais; Condições de trabalho; Trabalhadores; Restaurantes.

\begin{abstract}
The aim of this study was to analyze the prevalence of Work-Related Musculoskeletal Disorders (WMSD) in university restaurant workers. This is a cross-sectional and descriptive study with workers from four university restaurants in Belo Horizonte/MG. WMSD assessment in the last twelve months was investigated using the Nordic Musculoskeletal Symptoms Questionnaire (QNSO), composed of closed questions and organized by thematic sets that included demographic, socio-familial, health self-assessment, anthropometric variables, occupational characteristics and musculoskeletal symptoms. self-reported. Data were tabulated and analyzed by SPSS (p<0.05). 87 workers participated in the research $(72.5 \%$ of the sample), with a mean age of $37 \pm 9.8$ years, $81 \%$ female, mean working time of $9.3 \pm 7.5$ years, and working hours 40 hours per week. Regarding the presence of WMSD in the last twelve months, pain in the shoulder $(47.1 \%)$, lower back $(40.2 \%)$, ankles/feet $(39.1 \%)$, and temporal region $(37.1 \%)$ prevailed.). There was a prevalence of musculoskeletal symptoms associated with posture, depression, working hours and training courses. It is concluded that attention should be directed to the attitudes adopted by restaurant workers when performing tasks in order to reduce the incidence of occupational diseases.
\end{abstract}


Keywords: Musculoskeletal Disorders Related to Work (DORT); Occupational diseases; Work conditions; Workers; Restaurants.

\begin{abstract}
Resumen
El objetivo de este estudio fue analizar la prevalencia de Trastornos Musculoesqueléticas Relacionadas con el Trabajo (WMSD) en trabajadores de restaurantes universitarios. Se trata de un estudio descriptivo y transversal con trabajadores de cuatro restaurantes universitarios de Belo Horizonte / MG. La evaluación de WMSD en los últimos doce meses se investigó mediante el Cuestionario Nórdico de Síntomas Musculoesqueléticos (QNSO), compuesto por preguntas cerradas y organizadas por conjuntos temáticos que incluían demográficas, sociofamiliares, autoevaluación de salud, variables antropométricas, características ocupacionales y síntomas musculoesqueléticos. autoinformado. Los datos fueron tabulados y analizados por SPSS ( $p<0.05)$. En la investigación participaron 87 trabajadores $(72,5 \%$ de la muestra), con una edad media de $37 \pm 9,8$ años, $81 \%$ mujeres, jornada laboral media de 9,3 $\pm 7,5$ años y jornada laboral de 40 horas semanales. En cuanto a la presencia de WMSD en los últimos doce meses, predominó el dolor en hombro $(47,1 \%)$, espalda baja $(40,2 \%)$, tobillos / pies $(39,1 \%)$ y región temporal $(37,1 \%)$.). Hubo una prevalencia de síntomas musculoesqueléticos asociados con la postura, la depresión, las horas de trabajo y los cursos de capacitación. Se concluye que se debe prestar atención a las actitudes que adoptan los trabajadores de los restaurantes en el desempeño de sus tareas con el fin de reducir la incidencia de enfermedades profesionales.

Palabras clave: Trastornos Musculoesqueléticos Relacionados con el Trabajo (DORT); Enfermedades profesionales; Condiciones de trabajo; Trabajadores; Restaurantes.
\end{abstract}

\title{
1. Introdução
}

Nos últimos anos, em vários setores o mundo do trabalho tem sofrido intensas mudanças, as quais influenciam de forma significativa os modos de organização do trabalho, tais como: exigências por metas de produção, introdução de novas tecnologias, ritmo de trabalho acelerado, terceirizações e situações de empregabilidade instáveis, o que contribuem para o adoecimento de trabalhadores tanto no aspecto físico quanto mental (Antunes \& Praun, 2015).

As condições precárias de trabalho, os fatores físicos e psicossociais podem levar os trabalhadores a desenvolverem um quadro de insatisfações e fadiga, causando queda na produtividade, além de acidentes laborais e problemas de saúde.

De acordo com Antunes e Praun (2015, p. 423)

não se trata, portanto, de mero acaso que a maior incidência de casos de Lesões por Esforços Repetitivos/Distúrbios Osteomusculares Relacionados ao Trabalho (LER/DORT) e de transtornos mentais ocorra simultaneamente à disseminação em escala global dos processos de reorganização do trabalho e da produção e, de maneira articulada, à expansão das diferentes formas de precarização do trabalho, entre elas a expansão da terceirização.

Apesar de apresentarem nomenclaturas diferentes, LER e DORT são ocasionadas pelo mesmo fator: o uso intenso do sistema musculoesquelético no ambiente de trabalho (Cheung, Clemson, O'Loughlin \& Shuttleworth, 2016; Lasanen et al., 2017; Magas, Souza, Neves \& Nohama, 2019). Destaca-se que o DORT é caracterizado pela ocorrência de vários sintomas nas estruturas osteomioarticulares, predominantemente nos membros superiores, tais como: dor, parestesia, sensação de peso e fadiga. Esses sintomas surgem de forma insidiosa, concomitantes ou não, podem interagir entre si e devem ser sempre avaliados integralmente, tendo em vista o crescimento exponencial do DORT entre a população de trabalhadores (Ministério da Saúde, 2012, 2019).

De acordo com o Sistema Nacional de Atendimento Médico - SINAM, 134.670 casos de LER/DORT foram notificados entre 2007 e 2018. Neste período, o total de registros cresceu 506\%, passando de 3.433 casos, em 2007, para 17.392 em 2018 (Ministério da Saúde, 2019). O DORT se destaca no ranking de motivos que levam às faltas ao trabalho e à incapacidade de adultos trabalhadores no Brasil e em demais países industrializados (Assunção \& Abreu, 2017). É uma doença ocupacional que apresenta prejuízos econômicos para a sociedade e custos elevados de tratamento em vários países, sendo um dos maiores problemas de saúde pública no mundo (Brendbekken et al., 2016).

Nessa perspectiva, o setor de Alimentação Coletiva é um segmento do mercado de trabalho propício para o desenvolvimento dessas doenças ocupacionais, visto que a produção de refeições no Brasil convive com condições 
inadequadas de trabalho, problemas no ambiente e equipamentos, além de alta produtividade em um período de tempo relativamente curto. Esses e outros fatores contribuem para o desenvolvimento de problemas de saúde, acidentes de trabalho e insatisfação com horas extras (Oliveira \& Silva, 2016; Paes, Guilherme, Livera, Valle \& Silveira, 2020).

De modo geral, os locais destinados ao preparo das refeições apresentam condições físicas e ambientais inadequadas como ruído excessivo, temperatura elevada, iluminação deficiente, arranjo físico e instalações precárias. Além disso, as atividades realizadas nas Unidades de Alimentação e Nutrição caracterizam-se por movimentos repetitivos de membros superiores e coluna, levantamento de pesos excessivos e permanência na postura em pé por períodos prolongados de tempo (Brito, 2015; Wills, Davis \& Kotowski et al., 2013; Abreu, Spinelli \& Pinto, 2019).

Diante desse contexto, torna-se necessário identificar os fatores de risco que desencadeiam e ou agravam essas lesões. O diagnóstico dos DORT, muitas vezes, decorre de um quadro de queixa de dor intensa de uma pessoa que trabalha com movimentos repetitivos, mesmo que nos exames físicos, clínicos e de ultrassonografia não sejam encontradas lesões correspondentes à essa patologia (Hussain, Sivakumaran, Gill, Dhas, \& Ciurtin, 2018; Faisting \& Sato, 2019; Santos, Martins, Santos, Oliveira \& Brasileiro-Santos, 2021; Park, Lee \& Lee, 2021). O desenvolvimento desses distúrbios ocupacionais é multicausal e os fatores de risco são biomecânicos (ergonômicos), psicossociais relacionados à organização do trabalho e organizacionais (Costa, 2015).

Um estudo realizado com trabalhadores do setor de Alimentação Coletiva, localizado em Santos-SP, identificou que diversas questões de risco contribuíram para o surgimento de DORT, tais como: posturas e movimentos inadequados, manuseio de objetos pesados, ritmo acelerado, repetitividade de movimentos, problemas nas divisões das tarefas, deficiência nas pausas, espaço físico inadequados, número insuficiente de trabalhadores, ausência de conforto térmico, entre outros (Alencar, Cavalcanti \& Montrezor, 2013). Outro estudo realizado com 115 trabalhadores de um serviço de nutrição hospitalar do estado de São Paulo evidenciou que a prevalência de DORT foi de $71 \%$, sendo $81 \%$ em mulheres e de $32 \%$ nos homens (Jorge et al., 2009).

Assim, a justificativa para a realização do presente estudo foi a alta prevalência dos distúrbios osteomusculares DORT, encontrada nos trabalhadores de modo geral, entre eles o setor da Alimentação Coletiva.

Diante do exposto, o objetivo deste estudo foi analisar a prevalência de Distúrbios Osteomusculares Relacionadas ao Trabalho (DORT) em trabalhadores de restaurantes universitários.

\section{Metodologia}

Este é um estudo transversal, exploratório, quantitativo e descritivo (Pereira, Shitsuka, Parreira \& Shitsuka, 2018) realizado em população constituída por trabalhadores de quatro restaurantes universitários de Belo Horizonte, Minas Gerais, em 2017.

Para o cálculo amostral foram utilizados os critérios de Lwanga e Lemeshow (1991) para estimação de uma proporção para variáveis categóricas. Considerou-se a prevalência de dor em membros inferiores referida por $65,0 \%$ dos trabalhadores de um Serviço de Nutrição Hospitalar identificada conforme descrito em estudo de Isosaki, Cardoso, Glina, Alves e Rocha (2011), a nível de significância de 5\% ( $\mathrm{p}<0,05)$. Foi utilizado como critério de exclusão os contadores e operadores caixas dos restaurantes, para não criar um viés de dados.

Este estudo foi aprovado pelo Comitê de Ética em Pesquisas da Universidade Federal de Minas Gerais, sob parecer de $\mathrm{n}^{\mathrm{o}}$ 16227213.2.0000.5149 e todos os participantes assinaram o Termo de Consentimento Livre e Esclarecido antes de sua inclusão na amostra.

A coleta de dados foi realizada por estudantes capacitados para aplicar um questionário, de forma individual, aos trabalhadores, no seu local e horário de trabalho. Esse questionário era composto por questões fechadas, que incluíam variáveis 
demográficas (idade, sexo), sociofamiliares (escolaridade, estado conjugal, número de filhos, número de pessoas que moram na casa), autoavaliação de saúde, dados antropométricos (peso, altura e circunferência abdominal), características ocupacionais (tempo de trabalho e jornada média diária) e sintomas osteomusculares autorreferidos (Lima et al., 2016).

A avaliação de DORT nos últimos doze meses foi investigada com aplicação do Questionário Nórdico de Sintomas Osteomusculares (QNSO) (Isosaki, Cardoso, Glina, Alves \& Rocha, 2011). As partes investigadas foram região temporal, masseteres, região submandibular, laringe, parte anterior e posterior do pescoço, ombros, parte superior das costas, cotovelos, punhos ou mãos, parte inferior das costas, quadril ou coxas, joelhos, tornozelos ou pés (Isosaki, Cardoso, Glina, Alves \& Rocha, 2011).

O Índice de Massa Corporal (IMC) foi calculado a partir dos dados de peso e altura. Este índice é classificado para adultos em: "eutrofia" (valores entre 18,5 kg/m2 e 24,9 kg/m2), "sobrepeso" (valores entre $25 \mathrm{~kg} / \mathrm{m} 2$ e 29,9 kg/m2), e "obeso" (valores acima de $30 \mathrm{~kg} / \mathrm{m} 2$ ) (WHO, 2000). A classificação para circunferência da cintura (CC) foi feita de acordo com a Organização Mundial da Saúde (OMS), na qual valores acima de $80 \mathrm{~cm}$ para mulheres, e $94 \mathrm{~cm}$ para homens são considerados "risco elevado para Doenças Cardiovasculares (DCV)". O referencial utilizado para comparação da CC foi o proposto por Freedman et al. (1999).

Os dados foram tabulados e analisados com o apoio do software Statistical Package for the Social Sciences (SPSS) a um nível de significância de 5\% ( $<<0,05)$. Foi aplicado o teste qui-quadrado de Pearson, e alternativamente o teste exato de Fisher para amostras pequenas, para verificar se as associações encontradas apresentavam o nível de significância prefixado em 0,05 .

\section{Resultados}

Participaram do estudo 87 trabalhadores dos quatro restaurantes universitários (72,5\% da amostra). Na análise sociodemográfica a faixa etária média dos trabalhadores era de 33 anos e desvio padrão 8,86 anos; 62,0\% eram mulheres $(n=54)$, e 38,0\% homens $(n=33), 45,9 \%(n=40)$ casados, e a maioria se considerou de etnia parda $(57,0 \%, n=47)$.

Em relação à escolaridade, $48,0 \%(n=42)$ dos respondentes possuem ensino médio completo, $31,0 \%(n=27)$ ensino fundamental incompleto, sendo a maioria até o oitavo ano $(12,6 \% ; n=11)$. Em relação à renda, $63,2 \%$ (n=55) têm rendimento mensal até dois salários-mínimos e meio.

$\mathrm{Na}$ avaliação antropométrica a mediana do Índice de Massa Corporal (IMC) foi de 26,05 (19,9-39,8), com 42,2\% $(n=35)$ sendo eutróficos, ou seja, possuem uma alimentação de qualidade. A média para circunferência de cintura foi de 87,22 ( \pm DP 55-123). De acordo com a classificação da CC, 100,0\% das mulheres e dos homens não apresentaram risco elevado para Doenças Cardiovasculares (DCV).

Na Tabela 1 apresentam-se as características gerais sobre a saúde dos trabalhadores dos restaurantes universitários pesquisados. 
Tabela 1. Características gerais sobre a saúde de trabalhadores restaurantes universitários. Belo Horizonte, MG.

\begin{tabular}{|c|c|}
\hline Variáveis & Frequência \\
\hline Idade (anos, dp*) & $33 \pm 8,86$ \\
\hline \multicolumn{2}{|c|}{ Curso de capacitação: n (\%) } \\
\hline Sim & $49(56,3)$ \\
\hline Não & $38(43,7)$ \\
\hline \multicolumn{2}{|c|}{ Posturas que podem gerar dor: n (\%) } \\
\hline Nunca & $25(28,7)$ \\
\hline Raramente & $16(18,4)$ \\
\hline Às vezes & $34(39,1)$ \\
\hline Sempre & $12(13,8)$ \\
\hline \multicolumn{2}{|l|}{ IMC: $\mathbf{n}(\%)$} \\
\hline Eutrofia & $35(42,2)$ \\
\hline Sobrepeso & $29(34,9)$ \\
\hline Obesidade & $19(22,9)$ \\
\hline \multicolumn{2}{|c|}{ Doença ocupacional: n (\%) } \\
\hline Sim & $7(8,1)$ \\
\hline Não & $80(91,9)$ \\
\hline \multicolumn{2}{|l|}{ LER/DORT: n (\%) } \\
\hline Sim & (14) 16,1 \\
\hline Não & (73) 83,9 \\
\hline \multicolumn{2}{|c|}{ Acidente de trabalho: $\mathbf{n}(\%)$} \\
\hline Sim & $23(26,4)$ \\
\hline Não & $64(73,6)$ \\
\hline \multicolumn{2}{|l|}{ Hipertensão: n (\%) } \\
\hline Sim & $10(11,5)$ \\
\hline Não & $77(88,5)$ \\
\hline \multicolumn{2}{|l|}{ Diabetes n: (\%) } \\
\hline Sim & $4(4,6)$ \\
\hline Não & $83(95,4)$ \\
\hline \multicolumn{2}{|l|}{ Asma: n (\%) } \\
\hline Sim & $3(3,4)$ \\
\hline Não & $84(96,6)$ \\
\hline \multicolumn{2}{|l|}{ Depressão: n (\%) } \\
\hline Sim & $9(10,3)$ \\
\hline Não & $78(89,7)$ \\
\hline \multicolumn{2}{|c|}{ Transtorno de ansiedade: n (\%) } \\
\hline Sim & $4(4,6)$ \\
\hline Não & $83(95,4)$ \\
\hline
\end{tabular}

*dp: Desvio-padrão

Fonte: Elaborado pelos autores.

Quanto às informações gerais sobre saúde, 77,0\% $(\mathrm{n}=67)$ dos trabalhadores dos quatro restaurantes universitários informaram que nunca fizeram acompanhamento psicológico, 91,9\% $(n=80)$ nunca tiveram alguma doença ocupacional diagnosticada pelo médico, 73,6\% (n=64) nunca sofreram acidente de trabalho, 88,5\% (n=77) relataram não ter hipertensão, 
95,4\% ( $\mathrm{n}=83$ ) nunca tiveram diabetes; e 16,1\% tiveram DORT (Tabela 1).

A Tabela 2 apesenta as características ocupacionais dos trabalhadores.

Tabela 2. Características ocupacionais dos trabalhadores de restaurantes. Belo Horizonte, MG.

\begin{tabular}{lc}
\hline Variáveis & Frequência n (\%) \\
\hline Função & $2(2,3)$ \\
Auxiliar Serviços Gerais & $66(75,9)$ \\
Auxiliar de Cozinheiro & $1(1,1)$ \\
Auxiliar de Almoxarifado & $12(13,8)$ \\
Cozinheiro & $3(3,4)$ \\
Almoxarife & $2(2,3)$ \\
Técnico em Nutrição & $1(1,1)$ \\
Nutricionista & \\
Jornada de trabalho & $49(56,3)$ \\
8 horas & $38(43,7)$ \\
$12 / 36$ horas & \\
Curso de capacitação & $49(56,3)$ \\
Sim & $38(43,7)$ \\
não &
\end{tabular}

Fonte: Elaborado pelos autores.

As funções de maior frequência foram auxiliar de cozinheiro $(75,8 \% ; n=66)$, e cozinheiro $(13,7 \% ; n=12)$. A jornada de trabalho para a maioria dos colaboradores $(56,3 \% ; \mathrm{n}=49)$ foi de oito horas diárias, e os demais trabalhavam na escala 12/36 $(43,6 \% ; n=38)$. Além disto, 56,3\% $(n=49)$ dos trabalhadores dos restaurantes já realizaram um ou mais cursos de capacitação (Tabela 2).

A Tabela 3 apresenta a prevalência de DORT em relação aos segmentos corporais. 
Tabela 3. Prevalência de Distúrbios Osteomusculares Relacionados ao Trabalho - DORT, segundo segmento corporal referido. Belo Horizonte, MG.

\begin{tabular}{|c|c|c|}
\hline \multirow{2}{*}{ Segmento corporal referido } & \multicolumn{2}{|c|}{$\begin{array}{c}\text { DORT } \\
\text { SIM }\end{array}$} \\
\hline & $\mathbf{N}$ & $\%$ \\
\hline Em região (membros superiores ou inferiores) & 75 & 86,2 \\
\hline Pescoço, ombro ou parte alta do dorso & 71 & 81,6 \\
\hline Extremidades superiores distais (cotovelo, antebraço, punho, mão ou dedos) & 41 & 47,1 \\
\hline Membros inferiores (coxa, joelho, perna, tornozelo) & 80 & 91,9 \\
\hline Dor região anterior ao pescoço & 14 & 16,1 \\
\hline Dor abaixo do queixo & 2 & 2,3 \\
\hline Dor masseter & 2 & 2,3 \\
\hline Dor na região temporal & 33 & 37,9 \\
\hline Dor no pescoço & 30 & 34,5 \\
\hline Dor ombro & 41 & 47,1 \\
\hline Dor parte superior das costas & 32 & 36,8 \\
\hline Dor parte inferior das costas & 35 & 40,2 \\
\hline Dor cotovelos & 10 & 11,5 \\
\hline Dor punhos ou mãos & 31 & 35,6 \\
\hline Dor quadril ou coxas & 21 & 24,1 \\
\hline Dor joelhos & 25 & 28,7 \\
\hline Dor tornozelos ou pés & 34 & 39,1 \\
\hline
\end{tabular}

Fonte: Elaborado pelos autores.

Em relação à presença de sintomas osteomusculares nos últimos doze meses (Tabela 3), considerando-se as regiões corporais de forma isolada, prevaleceu a dor no ombro $(47,1 \% ; n=41)$, seguida pela parte inferior das costas $(40,2 \%$; $=35)$, tornozelos ou pés $(39,1 \% ; n=34)$, e região temporal $(37,1 \% ; n=33)$.

Analisando-se as regiões em conjunto verifica-se que 91,9\% dos indivíduos referiram DORT nos membros inferiores (coxa, joelho, perna, tornozelo), 81,6\% referiram DORT em pescoço, ombro ou parte alta do dorso, e 47,1\% em extremidades superiores distais (cotovelo, antebraço, punho, mãos) (Tabela 3). A prevalência dos DORT em pelo menos um segmento corporal foi de $86,2 \%$.

A seguir, encontram-se os resultados de associação, mediante análise estatística pelo teste do Qui-quadrado, entre a dor e os fatores associados a DORT, tais como depressão, transtorno de ansiedade, IMC, função, jornada de trabalho, cursos de capacitação e posturas adotadas (Tabelas 4 e 5 ). 
Research, Society and Development, v. 10, n. 7, e44210716965, 2021

(CC BY 4.0) | ISSN 2525-3409 | DOI: http://dx.doi.org/10.33448/rsd-v10i7.16965

Tabela 4 - Fatores associados à dor em trabalhadores de restaurantes institucionais de Belo Horizonte,MG.

\begin{tabular}{|c|c|c|c|c|c|c|c|c|c|c|c|c|}
\hline \multirow{3}{*}{ FATORES } & \multicolumn{12}{|c|}{ DOR POR SEGMENTO CORPORAL } \\
\hline & \multicolumn{2}{|c|}{ Região posterior pescoço } & \multicolumn{2}{|c|}{ Ombros } & \multicolumn{2}{|c|}{ Parte superior costas } & \multicolumn{2}{|c|}{ Cotovelos } & \multicolumn{2}{|c|}{ Punhos/mãos } & \multicolumn{2}{|c|}{ Região anterior pescoço } \\
\hline & $\operatorname{Sim}(\%)$ & Valor $\mathrm{p}^{*}$ & $\begin{array}{l}\text { Sim } \\
(\%)\end{array}$ & Valor $\mathrm{p}^{*}$ & $\operatorname{Sim}(\%)$ & Valor $\mathrm{p}^{*}$ & $\operatorname{Sim}(\%)$ & Valor p* & $\begin{array}{l}\text { Sim } \\
(\%)\end{array}$ & Valor $\mathrm{p}^{*}$ & $\operatorname{Sim}(\%)$ & Valor $\mathrm{p}^{*}$ \\
\hline Depressão & & & & & & & & & & & & \\
\hline Sim & 55,6 & \multirow{2}{*}{0,160} & 66,7 & \multirow{2}{*}{0,215} & 77,8 & \multirow{2}{*}{$\mathbf{0 , 0 1 1 *}$} & 44,4 & \multirow{2}{*}{$0,009 *$} & 66,7 & \multirow{2}{*}{0,063} & 22,2 & \multirow{2}{*}{0,633} \\
\hline Não & 32,1 & & 44,9 & & 32,1 & & 7,7 & & 32,1 & & 15,4 & \\
\hline Transtorno ansiedade & & & & & & & & & & & & \\
\hline Sim & 50,0 & \multirow{2}{*}{0,606} & 50,0 & \multirow{2}{*}{1,000} & 75,0 & \multirow{2}{*}{0,139} & 50,0 & \multirow{2}{*}{0,063} & 25,0 & \multirow{2}{*}{1,000} & 25,0 & \multirow{2}{*}{0,511} \\
\hline Não & 33,7 & & 47,0 & & 34,9 & & 9,6 & & 36,1 & & 215,7 & \\
\hline IMC & & & & & & & & & & & & \\
\hline Eutrófico & 34,3 & \multirow{3}{*}{0,847} & 45,7 & \multirow{3}{*}{0,599} & 34,3 & \multirow{3}{*}{0,753} & 8,6 & \multirow{3}{*}{0,901} & 37,1 & \multirow{3}{*}{0,720} & 20,0 & \multirow{3}{*}{0,283} \\
\hline Sobrepeso & 27,6 & & 51,7 & & 41,4 & & 6,9 & & 34,5 & & 6,9 & \\
\hline Obesidade & 31,6 & & 36,8 & & 31,6 & & 5,3 & & 26,3 & & 10,5 & \\
\hline \multicolumn{13}{|l|}{ Função } \\
\hline Auxiliar de serviços gerais & 50,0 & & 50,0 & & 100,0 & & 50,0 & & 0,0 & & 50,0 & \\
\hline Auxiliar de cozinheiro & 36,4 & & 53,0 & & 39,4 & & 10,6 & & 42,4 & & 18,2 & \\
\hline Auxiliar de almoxarifado & 100,0 & & 100,0 & & 100,0 & & 100,0 & & 100,0 & & 100,0 & \\
\hline Cozinheiro & 25,0 & 0,300 & 33,3 & 0,216 & 16,7 & 0,128 & 8,3 & 0,072 & 16,7 & 0,150 & 0 & 0,102 \\
\hline Almoxarife & 0,0 & & 0,0 & & 0,0 & & 0,0 & & 0,0 & & 0 & \\
\hline Técnico de nutrição & 0,0 & & 0,0 & & 50,0 & & 0,0 & & 0,0 & & 0 & \\
\hline Nutricionista & 100,0 & & 0,0 & & 0,0 & & 0,0 & & 0,0 & & 0 & \\
\hline Jornada de trabalho & & & & & & & & & & & & \\
\hline 8 horas & 40,8 & 0150 & 57,1 & $0024 \%$ & 42,9 & 0187 & 14,3 & 0502 & 46,9 & $0012 \%$ & 16,3 & 0046 \\
\hline $12 / 36$ horas & 26,3 & 0,158 & 34,2 & $0,034 *$ & 28,9 & 0,182 & 7,9 & 0,503 & 21,1 & $0,012^{*}$ & 15,8 & 0,940 \\
\hline Cursos de capacitação & & & & & & & & & & & & \\
\hline Sim & 22,4 & $0007 *$ & 40,8 & & 32,7 & & 10,2 & & 26,5 & & 12,2 & \\
\hline Não & 50,0 & $0,007^{*}$ & 55,3 & 0,181 & 42,1 & 0,364 & 13,2 & 0,668 & 47,4 & $0,044^{*}$ & 21,1 & 0,267 \\
\hline $\begin{array}{l}\text { Adotar postura que pode ger } \\
\text { dor/desconforto no trabalhe }\end{array}$ & & & & & & & & & & & & \\
\hline Nunca & $8,0 \mathrm{a}$ & & $12,0 \mathrm{a}$ & & $12,0 \mathrm{a}$ & & 0,0 & & $12,0 \mathrm{a}$ & & 0,0 & \\
\hline Raramente & $31,3 \mathrm{a}$ & & $50,0 \mathrm{a}$ & & $31,3 \mathrm{a}$ & $0002 *$ & 12,5 & 0184 & $43,8 \mathrm{a}$ & $0000 *$ & 25,0 & 0074 \\
\hline Às vezes & $44,1 \mathrm{~b}$ & $0,002^{*}$ & $61,8 \mathrm{~b}$ & $<0,001^{*}$ & $44,1 \mathrm{a}$ & $0,002^{*}$ & 17,6 & 0,184 & $38,2 \mathrm{a}$ & $0,009^{*}$ & 20,6 & $0,0 / 4$ \\
\hline Sempre & $66,7 \mathrm{c}$ & & $75,0 \mathrm{c}$ & & $75,0 \mathrm{~b}$ & & 16,7 & & $66,7 \mathrm{~b}$ & & 25,0 & \\
\hline
\end{tabular}

Nota: Frequências seguidas pela mesma letra na coluna, não diferem significativamente pelo teste Bonferroni ( $p>0,05)$. "Valor $\mathrm{p}$ no teste qui-quadrado. Fonte: Autores. 
Research, Society and Development, v. 10, n. 7, e44210716965, 2021

(CC BY 4.0) | ISSN 2525-3409 | DOI: http://dx.doi.org/10.33448/rsd-v10i7.16965

Tabela 5 - Fatores associados à dor em trabalhadores de restaurantes institucionais de Belo Horizonte, MG.

\begin{tabular}{|c|c|c|c|c|c|c|c|c|c|c|c|c|}
\hline \multirow{3}{*}{ FATORES } & \multicolumn{12}{|c|}{ DOR POR SEGMENTO CORPORAL } \\
\hline & \multicolumn{2}{|c|}{$\begin{array}{c}\text { Parte inferior das } \\
\text { costas }\end{array}$} & \multicolumn{2}{|c|}{ Quadril/coxas } & \multicolumn{2}{|c|}{$\begin{array}{c}\text { Região abaixo do } \\
\text { queixo }\end{array}$} & \multicolumn{2}{|c|}{ Masseter, bochechas } & \multicolumn{2}{|c|}{ Joelhos } & \multicolumn{2}{|c|}{ Tornozelos/Pés } \\
\hline & $\operatorname{Sim}(\%)$ & Valor $\mathrm{p}^{*}$ & $\begin{array}{l}\text { Sim } \\
(\%)\end{array}$ & Valor $\mathrm{p}^{*}$ & $\operatorname{Sim}(\%)$ & Valor $\mathrm{p}^{*}$ & $\operatorname{Sim}(\%)$ & Valor $\mathrm{p}^{*}$ & $\operatorname{Sim}(\%)$ & Valor $\mathrm{p}^{*}$ & $\operatorname{Sim}(\%)$ & Valor $\mathrm{p}^{*}$ \\
\hline \multicolumn{13}{|l|}{ Depressão } \\
\hline Sim & 44,4 & \multirow{2}{*}{1,000} & 55,6 & \multirow{2}{*}{$\mathbf{0 , 0 3 4 *}$} & 0,0 & \multirow{2}{*}{1,000} & 0,0 & \multirow{2}{*}{1,000} & 44,4 & \multirow{2}{*}{0,272} & 55,6 & \multirow[b]{2}{*}{0,304} \\
\hline Não & 39,7 & & 20,5 & & 2,6 & & 2,6 & & 26,9 & & 37,2 & \\
\hline \multicolumn{13}{|l|}{ Transtorno de ansiedade } \\
\hline Sim & 25,0 & \multirow{2}{*}{0,646} & 50,0 & \multirow{2}{*}{0,244} & 0,0 & \multirow{2}{*}{1,000} & 0,0 & \multirow{2}{*}{1,000} & 25,0 & \multirow{2}{*}{1,000} & 75,0 & \multirow{2}{*}{0,295} \\
\hline Não & 41,0 & & 22,9 & & 2,4 & & 2,4 & & 28,9 & & 37,3 & \\
\hline \multicolumn{13}{|l|}{ IMC } \\
\hline Eutrófico & 42,9 & \multirow{3}{*}{0,587} & 20,0 & \multirow{3}{*}{0,995} & 0,0 & \multirow{3}{*}{ NA } & 0,0 & \multirow{3}{*}{ NA } & 22,9 & & 34,3 & \\
\hline Sobrepeso & 31,0 & & 20,7 & & 0,0 & & 0,0 & & 37,9 & 0,310 & 41,4 & 0,842 \\
\hline Obesidade & 42,1 & & 21,1 & & 0,0 & & 0,0 & & 21,1 & & 36,8 & \\
\hline Função & & & & & & & & & & & & \\
\hline Auxiliar serviços gerais & 50,0 & & 50,0 & & $0,0 \mathrm{a}$ & & $0,0 \mathrm{a}$ & & 50,0 & & 100,0 & \\
\hline Auxiliar cozinheiro & 42,4 & & 21,2 & & $1,5 \mathrm{a}$ & & $1,5 \mathrm{a}$ & & 33,3 & & 36,4 & \\
\hline Auxiliar almoxarifado & 100,0 & & 100,0 & & $100,0 \mathrm{a}$ & & $100,0 \mathrm{a}$ & & 100,0 & & 100,0 & \\
\hline Cozinheiro & 33,3 & 0,675 & 25,0 & 0,497 & $0,0 \mathrm{a}$ & $<0,001^{*}$ & $0,0 \mathrm{a}$ & $<0,001^{*}$ & 8,3 & 0,206 & 33,3 & 0,345 \\
\hline Almoxarife & 33,3 & & 33,3 & & $0,0 \mathrm{a}$ & & $0,0 \mathrm{a}$ & & 0,0 & & 33,3 & \\
\hline Técnico nutrição & 0,0 & & 50,0 & & $0,0 \mathrm{a}$ & & $0,0 \mathrm{a}$ & & 0,0 & & 50,0 & \\
\hline Nutricionista & 0,0 & & 0,0 & & $0,0 \mathrm{a}$ & & $0,0 \mathrm{a}$ & & 0,0 & & 100,0 & \\
\hline Jornada de trabalho & & & & & & & & & & & & \\
\hline 8 horas & 42,9 & $0-570$ & 28,6 & & 4,1 & 0500 & 4,1 & $0-509$ & 32,7 & 0250 & 38,8 & 0017 \\
\hline $12 / 36$ horas & 36,8 & $0,5 / 0$ & 18,4 & $0,2 / 2$ & 0,0 & 0,502 & 0,0 & 0,502 & 23,7 & 0,359 & 39,5 & $0,94 /$ \\
\hline Cursos de capacitação & & & & & & & & & & & & \\
\hline Sim & 28,6 & & 16,3 & & 0,0 & & 0,0 & & 20,4 & & 32,7 & 0.163 \\
\hline Não & 55,3 & $0,012^{*}$ & 34,2 & 0,053 & 5,3 & 0,188 & 5,3 & 0,188 & 39,5 & 0,051 & 47,4 & 0,163 \\
\hline $\begin{array}{l}\text { Adotar postura que pode g } \\
\text { dor/desconforto no trabal }\end{array}$ & & & & & & & & & & & & \\
\hline Nunca & $16,0 \mathrm{a}$ & & $4,0 \mathrm{a}$ & & $0,0 \mathrm{a}$ & & $0,0 \mathrm{a}$ & & 20,0 & & 20,0 & \\
\hline Raramente & $43,8 \mathrm{a}$ & $0006 *$ & $25,0 \mathrm{a}$ & $0015 *$ & $12,5 \mathrm{a}$ & $0028 *$ & $12,5 \mathrm{a}$ & $0028 *$ & 37,5 & 0458 & 37,5 & 0105 \\
\hline Às vezes & $44,1 \mathrm{a}$ & $0,006^{*}$ & $29,4 \mathrm{a}$ & $0,010^{*}$ & $0,0 \mathrm{a}$ & $0,028^{*}$ & $0,0 \mathrm{a}$ & $0,028^{*}$ & 26,5 & 0,458 & 50,0 & 0,100 \\
\hline Sempre & $75,0 \mathrm{~b}$ & & $50,0 \mathrm{~b}$ & & $0,0 \mathrm{a}$ & & $0,0 \mathrm{a}$ & & 41,7 & & 50,0 & \\
\hline
\end{tabular}

Nota: Frequências seguidas pela mesma letra na coluna, não diferem significativamente pelo teste Bonferroni ( $\mathrm{p}>0,05)$. "Valor $\mathrm{p}$ no teste qui-quadrado. Fonte: Autores. 
Nos dados relativos à associação entre dor, como um sintoma característico do DORT, e adoção de posturas que podem gerar dores ou desconforto (Tabela 4), constatou-se uma diferença estatisticamente significativa entre trabalhadores que sempre têm dores ou desconforto no quadril ou coxas (50,0\% vs 4,0\%; p=0,002), regiões do punhos ou mãos $(66,7 \%$ vs $12,0 \%$; $\mathrm{p}=0,001)$, parte superior das costas $(75,0 \%$ vs $12,0 \%$; $\mathrm{p}<0,001)$, parte inferior das costas $(75,0 \%$ vs $16,0 \%$; $\mathrm{p}=0,001)$, e aqueles trabalhadores que nunca adotam posturas que podem gerar dores ou desconforto no trabalho ou fora do trabalho.

A queixa de dor no ombro e pescoço foi mais frequente entre aqueles que mencionaram que sempre durante o seu horário de trabalho adotam posturas que podem gerar dores ou desconforto muscular, do que os que mencionaram nunca adotam posturas que podem gerar dores ou desconforto muscular: $75,0 \%$ vs $12,0 \%$; p<0,001 e $(66,7 \%$ vs $8,0 \%$; p $<0,001)$, respectivamente. Dor no ombro e pescoço também foi mais frequente entre aqueles que mencionaram que às vezes durante o seu horário de trabalho adotam posturas que podem gerar dores ou desconforto muscular no trabalho.

Pôde-se também observar uma associação significativa para dor nos punhos ou mãos $(57,1 \%$; $=0,012)$ e no ombro $(46,9 \% ; p=0,034)$ entre aqueles trabalhadores de restaurantes com jornada de trabalho de 8 horas.

\section{Discussão}

No presente estudo a maioria dos trabalhadores era do sexo feminino (62,0\%). De maneira semelhante Isosaki, Cardoso, Glina, Alves e Rocha (2011) encontraram em serviços de nutrição de dois hospitais brasileiros, localizados em São Paulo (SP), uma maior presença de trabalhadores do sexo feminino, 86\% em um hospital público (n=123) e 67\% em um hospital privado $(\mathrm{n}=162)$. Casarotto e Mendes (2003) também identificaram predominância feminina (74\%) em um estudo realizado em quatro restaurantes universitários e um restaurante de um hospital pediátrico (n=256).

Verificou-se uma prevalência de $16,1 \%$ de DORT na população estudada (Tabela 1). Este achado corrobora os resultados de outros estudos que demonstraram valores semelhantes de DORT para trabalhadores de restaurantes. Aguiar, Valente e Fonseca (2010), por exemplo, constataram que 15\% dos trabalhadores de restaurantes populares tiveram diagnóstico de DORT, enquanto Casarotto e Mendes (2003), verificaram que 14,6\% dos trabalhadores de restaurantes universitários e de hospital apresentavam DORT.

Em relação à região do corpo sobre a qual os trabalhadores desta pesquisa apresentaram maiores queixas destacam-se a dor no ombro $(47,1 \%$; $n=41)$, seguida da parte inferior das costas $(40,2 \%$; $n=35)$, tornozelos ou pés $(39,1 \%$; $n=34)$, e região temporal $(37,1 \% ; n=33)$.

Geralmente, as atividades realizadas pela maioria dos trabalhadores em restaurantes envolvem levantamento de carga, realização de movimentos contínuos e repetitivos, permanência prolongada em pé e outras posturas inadequadas (Wills, Davis \& Kotowski, 2013), por isto, as articulações e membros superiores e inferiores são tão afetados, além da coluna vertebral, quadril e coxas.

Para Casarotto e Mendes (2003), os fatores de risco responsáveis pelo aparecimento dos distúrbios osteomusculares, incluindo lesões no ombro, presentes em todas as atividades desenvolvidas nas cozinhas são provenientes de movimentos inadequados para membros superiores e força manual excessiva.

Segundo Haukka et al. (2006), a maior prevalência, em três meses, de dores osteomusculares apontada por 495 cozinheiras do gênero feminino foram em regiões da coluna cervical, da coluna lombar e dos punhos/mãos, sendo que 73,0\% tiveram dores em pelo menos duas delas. Em um estudo realizado em Santos-SP, os pesquisadores observaram que as principais regiões acometidas pelos DORT foram punhos/mãos, ombros e quadril/coxas. Estes autores relataram, ainda, que a presença de sintomas osteomusculares entre as trabalhadoras, tanto nos últimos 12 meses, quanto nos últimos 30 e 7 dias, gera 
preocupação quanto ao possível agravamento dos quadros de saúde (Alencar, Cavalcanti \& Montrezor, 2013).

As dores e o adoecimento causado têm consequências diretas na assiduidade e produtividade do trabalhador. Isosaki, Cardoso, Glina, Alves e Rocha (2011) encontraram como causa principal do absenteísmo entre trabalhadores de Serviços de Nutrição e Dietética de dois hospitais em São Paulo, doenças relacionadas às atividades exercidas pelos trabalhadores e, dentre essas, as do sistema osteomuscular e do tecido conjuntivo.

De acordo com os resultados obtidos no presente estudo foi possível identificar a prevalência de Sintomas Osteomusculares Relacionados ao Trabalho - DORT, segundo segmento corporal (Tabela 3), e associados às posturas inadequadas, depressão emocional, jornada de trabalho, função e realização de cursos de capacitação (Tabelas 4 e 5 ).

Os principais determinantes para o surgimento das doenças osteomusculares são questões relacionadas à postura, ao esforço físico e aos fatores ambientais. Os trabalhadores vivenciam limitações laborais que podem levar a quadros de tristeza e insatisfação, tanto pela capacidade de trabalho diminuída, quanto pela ineficácia dos tratamentos realizados, quando não há alteração no processo e na organização do trabalho (Oliveira \& Almeida, 2017).

A dor é uma das principais causas do sofrimento humano, que acaba provocando disfunções físicas e psicossociais no indivíduo, diminuindo desta forma sua qualidade de vida, o que reflete no desempenho do trabalho do indivíduo e em suas relações sociais e familiares. Portanto, sempre que a queixa de dor aparecer ela deve ser avaliada por uma equipe multiprofissional de saúde, para que se seja traçado um cuidado individualizado, dirigido à causa desencadeante da dor, a fim de eliminá-la (Lopes, 2001; Antunes \& Praun, 2015).

Estudos de Sticca, Mandarini e Silva (2019), Alencar e Merlo (2018), Brito (2015), Isosaki et al. (2011), mostraram que os fatores de risco para LER/DORT podem ser relacionados por exemplo com: a organização do trabalho, o grau de adequação do posto de trabalho; a exposição a vibrações e ao frio; as pressões mecânicas localizada nos tecidos; as posturas inadequadas e as cargas mecânicas musculoesqueléticas. Ainda de acordo com os estudos citados, dentre os fatores relacionados com as cargas mecânicas musculoesqueléticas encontram-se: a força; a repetitividade; a duração da carga; o tipo de preensão; a postura; e o método de trabalho. Outro estudo realizado com trabalhadores de um restaurante de São Paulo também identificou fatores como posturas inadequadas e realização de esforço repetitivo durante a execução das tarefas (Gadotti, Silva \& Andrade, 2015).

Na Austrália foi realizado um estudo em uma Instituição de Longa Permanência para o cuidado de idosos, em que a maior parte dos trabalhadores desconhecia quais riscos psicossociais relacionados ao trabalho afetavam a sua saúde musculoesquelética e mental (Oakman, Macdonald \& Kinsman, 2019). A falta de conhecimento desses fatores pode dificultar a busca por uma solução e prolongar ainda mais o sofrimento.

Da mesma forma, em um trabalho realizado por Sticca, Mandarini e Silva (2019) com trabalhadores em um restaurante universitário foi observado que os fatores de risco ergonômicos e psicossociais relacionados ao trabalho, ou seja, o ambiente físico, a carga de trabalho elevada e as dificuldades nas relações socioprofissionais, podem ser consideradas variáveis indissociadas que interferem na saúde física e mental do trabalhador.

Brito (2015) em uma Unidade de Alimentação e Nutrição Hospitalar também apontou diversos problemas de ambiência dos postos de trabalho, tanto em relação à infraestrutura quanto à organização do trabalho. Conforme alertam os autores mencionados, estas inconformidades podem resultar em um ambiente favorável ao adoecimento e riscos de acidentes, além de comprometer a qualidade do alimento produzido.

Isosaki, Cardoso, Glina, Alves e Rocha (2011) identificaram que as jornadas diárias prolongadas de trabalho (de 8 a 12 horas diárias), sem a realização das pausas, foi a principal causa da alta prevalência de sintomas osteomusculares nos membros inferiores de $70 \%$ dos trabalhadores. Em outros países foram encontrados resultados semelhantes como os 
encontrados por Park, Lee e Lee (2021) em um estudo realizado com 1909 trabalhadores de restaurantes na Coreia, que identificaram a necessidade de pausas de descanso como uma intervenção no local de trabalho para prevenir os DORT. Laperriere, Messing e Bourbonnais (2017) também concluíram que trabalhadores em três restaurantes localizados na cidade de Quebec no Canadá, expostos a um risco elevado de DORT, na maior parte das vezes escolhiam o bem-estar dos clientes, o aumento da produtividade e a qualidade do serviço em detrimento à sua saúde.

Chang et al. (2020) e Oakman, Macdonald e Kinsman (2019), alertam que a falta de consciência dos riscos a que são submetidos os trabalhadores pode dificultar a busca por uma solução, prolongar ainda mais o sofrimento e comprometer a capacidade de trabalho, portanto, o desenvolvimento de estratégias que visam mudanças nas condições de trabalho é essencial para a melhoria da Qualidades de Vida (QV) dos trabalhadores.

Segundo Bartilotti et al. (2009), o número de afastamentos do trabalho devido a transtornos mentais relacionados à atividade laboral tem sido crescente no Brasil, e pode ser um dos fatores relativos ao agravamento dos quadros clínicos dos casos de LER/DORT. Os autores afirmam também que apesar da alta prevalência de sintomas mentais associados ao adoecimento crônico musculoesquelético (sintomas de ansiedade e depressivos), transtornos mentais relacionados ao trabalho podem se apresentar como o agravo mais importante ou mesmo prioritário, por isto merecem cuidados específicos.

No presente estudo, a jornada de trabalho também foi associada a dores no ombro e punhos/mãos. Verificou-se adicionalmente que a realização de Cursos de Capacitação foi determinante em não resultar em dores em algumas regiões do corpo. Oliveira e Silva (2016) corroboram esse entendimento, afirmando que as capacitações com enfoque prático são medidas muito eficazes para a transmissão de conhecimentos aos trabalhadores de restaurantes, levando a correção das posturas e movimentos corporais.

A produção de refeições envolve inúmeros fatores, tais como o número de operadores, o tipo de alimento utilizado, as técnicas de preparo e infraestrutura, exigindo equipamentos e utensílios que visam otimizar as operações, tornando-as mais rápidas e confiáveis do ponto de vista da conformidade do produto final (Abreu, Spinelli \& Pinto, 2019). Ao realizar a correlação com os fatores de risco no trabalho, dividido em fatores de risco ambientais e os fatores de riscos das operações, verifica-se que os trabalhadores de restaurantes frequentemente estão em contato com todos os fatores mencionados, e o que se associa a eles, como ruídos, vibrações, calor, quedas, levantamento de peso, repetitividade e posturas inadequadas de trabalho.

As doenças crônicas decorrentes de trabalhos desenvolvidos nessas condições exigem um tratamento permanente, e algumas estratégias podem ser adotadas a partir de mudanças na organização do trabalho, como, por exemplo, a realização de pausas durante a jornada, a flexibilização das cobranças por produtividade e a diminuição de horas extras (Zavarizzi, Carvalho \& Alencar, 2019).

Em uma pesquisa cujo objetivo foi compreender as relações entre os aspectos da organização do trabalho e a saúde de atendentes de nutrição acometidos por LER/ DORT, Alencar e Merlo (2018) constataram que modos de organização do trabalho e gestão com cobranças excessivas, assédio moral e falta de reconhecimento no trabalho geram sofrimento e influenciam nos processos de adoecimento.

De acordo com Dale e Dias (2018), o corpo, que deveria ser um meio utilizado para produção e realização de atividades prazerosas e construtivas, tanto do ponto de vista objetivo quanto subjetivo, está sendo obrigado cada dia mais - e por necessidades diversas - a se submeter a trabalhos desgastantes, que podem impedi-lo ou limitá-lo, pela doença, de realizar tarefas profissionais e pessoais.

No mundo capitalista, o trabalhador perde o poder e a autonomia sobre o seu próprio corpo, sem os subsídios necessários para cumprir suas atividades de forma adequada e confortável tendo que se submeter a atividades que, muitas vezes, favorecem o adoecimento do seu corpo e ainda oprimem a sua singularidade e a capacidade de criação (Dale \& Dias, 
Research, Society and Development, v. 10, n. 7, e44210716965, 2021

(CC BY 4.0) | ISSN 2525-3409 | DOI: http://dx.doi.org/10.33448/rsd-v10i7.16965

2018). Consideram-se como limitações do presente estudo a baixa validade externa, por ter sido realizado em uma amostra de conveniência. Por outro lado, têm-se como pontos fortes os cuidados metodológicos de coleta de dados para que os resultados expressassem de fato a realidade vivenciada pelos trabalhadores dos restaurantes universitários pesquisados.

\section{Conclusão}

Os dados deste estudo contribuíram para a avaliação distúrbios osteomusculares relacionados ao trabalho no setor de Alimentação Coletiva e demonstram a existência e impacto destes distúrbios na saúde dos trabalhadores de restaurantes universitários.

Constatou-se que as causas do surgimento dos DORT são múltiplas, originadas de fatores isolados, conjuntos (fatores biomecânicos, organizacionais e psicossociais) e multidimensional (dimensão individual, grupal e social), mas que exercem seus efeitos de forma simultânea e interligada. Ao se compreender os mecanismos dessa multicausalidade, percebe-se a necessidade da abordagem global para se prevenir os DORT.

Desta forma, maior atenção deve ser direcionada à ambiência dos locais de trabalho, às posturas adotadas pelos trabalhadores na execução das tarefas e a sua saúde mental, a fim de reduzir a incidência de doenças ocupacionais. Além disso, os trabalhadores com dor osteomuscular precisam estar conscientes que a sua presença sinaliza para o desgaste e o adoecimento. Nesse sentido, necessitam auxiliar na construção das estratégias que possam precocemente evitar seu agravamento. Ressalta-se, portanto, o papel do gestor do restaurante na condução das atividades a frente da equipe, bem como em proporcionar condições gerenciais e ambientais adequadas para execução das atividades laborais a fim de favorecer conforto e saúde ao trabalhador.

Sugere-se que outros estudos possam ser realizados com diferentes tipos de restaurantes como comerciais e hospitalares, de forma a proporcionar um maior conhecimento sobre a ocorrência dos DORT no setor de Alimentação Coletiva, e implantar estratégias para reduzir os impactos destes distúrbios na saúde dos trabalhadores.

\section{Referências}

Abreu, E. S., Spinelli, M. G. N., \& Pinto, A. M. S. (2019). Gestão de unidades de alimentação e nutrição: um modo de fazer. Editora Metha.

Aguiar, O. B. D., Valente, J. G., \& Fonseca, M. D. J. M. D. (2010). Descrição sociodemográfica, laboral e de saúde dos trabalhadores do setor de serviços de alimentação dos restaurantes populares do estado do Rio de Janeiro. Revista de Nutrição, 23(6), 969-982. http://dx.doi.org/10.1590/S141552732010000600004 .

Alencar, M. C. B., Cavalcanti, T. A., \& Montrezor J.B. (2013). Condições de trabalho em uma cozinha industrial e distúrbios osteomusculares de trabalhadores. Cadernos de Terapia Ocupacional da UFSCar, 21(1), 155-162. http://dx.doi.org/10.4322/cto.2013.020

Alencar, M. C. B., \& Merlo, A. R. C. (2018). A saúde em troca da excelência: o sofrimento de atendentes de nutrição de um hospital público acometidos por LER/DORT. Saúde e Sociedade, 27(1), 215-226. http://dx.doi.org/10.1590/s0104-12902018170873

Antunes, R. \& Praun, L. A (2015). Sociedade dos adoecimentos no trabalho. Serviço Social e Sociedade, n. 123, p. 407-427. https://doi.org/10.1590/01016628.030

Assunção, A. A., \& Abreu, M. N. S. (2017). Fatores associados a distúrbios osteomusculares relacionados ao trabalho autorreferidos em adultos brasileiros. Revista de Saúde Pública, 51 Supl 1:10s. https://doi.org/10.1590/s1518-8787.2017051000282

Bartilotti, C. B., de Andrade, P. R., de Mattos Varandas, J., Ferreira, P. C. G., \& Cabral, C. (2009). Programa de Reabilitação Ampliada (PRA): uma abordagem multidimensional do processo de reabilitação profissional. Acta Fisiátrica, 16(2), 66-75. https://www.revistas.usp.br/actafisiat rica/article/view/103169

Brendbekken, R., Eriksen, H. R. , Grasdal, A., Harris, A., Hagen, E. M., \& Tangen, T. (2016). Return to work in patients with chronic musculoskeletal pain: multidisciplinary intervention versus brief intervention: a randomized clinical trial. Journal of Occupational Rehabilitation, 26(2), 1-10. 10.1007 / s10926$016-9634-5$

Brito, K. M. F. de. (2015). Riscos à saúde dos trabalhadores em unidade de alimentação e nutrição de um Hospital Universitário. Dissertação (Metrado). Universidade Federal do Rio Grande do Norte. https://repositorio.ufrn.br/bitstream/123456789/20529/1/KatiaMariaFernandesDeBrito_DISSERT.pdf 
Research, Society and Development, v. 10, n. 7, e44210716965, 2021

(CC BY 4.0) | ISSN 2525-3409 | DOI: http://dx.doi.org/10.33448/rsd-v10i7.16965

Casarotto, R. A. \& Mendes, L. F. (2003). Queixas, doenças ocupacionais e acidentes de trabalho em trabalhadores de cozinhas industriais. Revista Brasileira de Saúde Ocupacional, 28(107-108), 119-126. https://doi.org/10.1590/S0303-76572003000200011.

Costa, G. T. V. (2015). Fatores de Risco dos Distúrbios Osteomusculares Relacionados ao Trabalho em Trabalhadores de Enfermagem: Revisão Integrativa. Dissertação (Mestrado). Universidade Federal do Rio de Janeiro. http://objdig.ufrj.br/51/teses/837961.pdf

Chang, Y-F., Yeh, C-M., Huang, S-H., C-C Ho, C-C, Li, R-H., Wang, W-H., Tang F-C. (2020). Work ability and quality of life in patients with work-related musculoskeletal disorders. International Journal Environmental Research Public Health, 17, 1-12. https://doi.org/10.3390/ijerph17093310

Cheung TW, Clemson L, O'Loughlin K, Shuttleworth R. (2016). Understanding decision-making towards housework among women with upper limb repetitive strain injury. Australian Occupational Therapy Journal, 63(1), 37-46. 10.1111 / 1440-1630.12254

Dale, A. P. \& Dias, M. D. do A. A. (2018). A extravagância de trabalhar doente: o corpo no trabalho em indivíduos com diagnóstico de LER/DORT. Trabalho, Educação e Saúde, 16(1), 263-282. http://dx.doi.org/10.1590/1981-7746-sol00106

Faisting, A. L. R. F. \& Sato, T. O. (2019). Effectiveness of ergonomic training to reduce physical demands and musculoskeletal symptoms - An overview of systematic reviews. Nternational. Journal of Industrial Ergonomics, 74, 102845. https://doi.org/10.1016/j.ergon.2019.102845

Freedman, D. S., Serdula, M. K., Srinivasan, S. R., \& Berenson, G. S. (1999) Relation of circumference and skinfold thicknesses to lipid and insulin concentrations in children and adolescents: The Bogalusa Heart Study. The American Journal Clinical Nutrition, 69, 308-3017. https://doi.org/10.1093 / ajcn / 69.2 .308

Gadotti, T., Silva, Z. M., Andrade, A. C. et al. (2015). A importância da ergonomia no processo de melhoria das condições de trabalho no setor de alimentação coletiva: fatores envolvidos no desenvolvimento de sintomas osteomusculares. Higiene Alimentar, 29, 242-243. https://higienealimentar.com.br/wpcontent/uploads/2019/07/242-243.pdf

Gaulejac, V. Gestão como doença social: ideologia, poder gerencialista e fragmentação social. Aparecida: Ideias \& Letras, 2017.

Haukka, E., Leino-Arjas, P., Solovieva, S., Ranta, R., Viikari-Juntura, E. \& Riihimäkiet, H. (2006). Co-occurance of musculoskeletal pain among female kitchen workers. International Archives of Arch Occupational Environmental Health, Berlin, 80(2),141-148. https://doi.org/10.1007/s00420-006-0113-8

Hussain, S., Sivakumaran, P., Gill, A., Dhas, D. \& Ciurtin, C. (2018). Ultrasonography- detected subclinical inflammation in patients with hand osteoarthri- tis and established rheumatoid arthritis: a comparison between two different pathologies using the same ultrasound examination proto- col. Musculoskeletal Care, 6(1), 26-31. https://doi.org/10. 1002/msc.1197.

Isosaki, M., Cardoso, E., Glina, D. M. R., Alves, A. C. C, Rocha, L. E. (2011). Prevalência de sintomas osteomusculares entre trabalhadores de um Serviço de Nutrição Hospitalar em São Paulo. Revista Brasileira de Saúde Ocupacional, 36(124), 238-46. https://doi.org/10.1590/S0303-76572011000200007 .

Jorge, A. T., Glina, D. M. R., Iosaki M., Ribeiro, A. C DI C. A., Ferreira Junior, M., Rocha, L. E. (2009). Work related musculoskeletal disorders: risk factors among hospital food service workers. Revista Brasileira de Medicina do Trabalho, 7(1), 2-10. http://www.rbmt.org.br/details/140/pt-br/disturbiososteomusculares-do-trabalho--fatores-de-risco-em-trabalhadores-de-nutricao-hospitalar

Lasanen, R., Malo, M. K. H., Airaksinen, O., Karhu, J., Toyras, J., Julkunen, P. (2017). infrared thermography reveals effect of working posture on skin temperature in office workers. International Journal of Occupational Safety and Ergonomics, 24, 457-463. $10.1080 / 10803548.2017 .1336299$

Laperriere, E., Messing, K., \& Bourbonnais, R. (2017). Work activity in food service: the significance of customer relations, tipping practices and gender for preventing musculoskeletal disorders. Applied Ergonomics, 58, 89e101. https://doi.org/10.1016/j.apergo.2016.05.013

Lima, D. P., Sties, S. W., Gonzales, A. I. et al. (2016). Questionário para avaliação da dor muscoloesqulética em praticantes de exercícios (Q-ADOM). Revista Brasileira de Medicina do Esporte, 22(5), 374-380. https://doi.org/10.1590/1517-869220162205161347.

Lopes, M. J. M. (2001). A saúde das trabalhadoras da saúde: algumas questões. In: Haag, G. S., Lopes, M. J. M., Schuck, J. S. A enfermagem e a saúde dos trabalhadores. $2^{\mathrm{a}}$ ed. Goiânia: Cultura e Qualidade; 2001.

Lwanga, S. K. \& Lemeshow, S. Sample Size Determination in Health Studies. Geneva: World Health Organization, 1991.

Magas, V., de Souza, M. A., Neves, E. B., \& Nohama, P. (2019). Evaluation of thermal imaging for the diagnosis of repetitive strain injuries of the wrist and hand joints. Research on Biomedical Engineering, 35(1), 57-64. https://doi.org/10.1007/s42600-019-00009-y

Matos, C. H. \& Proença, R. P. C. (2003). Condições de trabalho e estado nutricional de operadores do setor de alimentação coletiva: um estudo de caso. Revista de Nutrição, 16(4), 493-502. https://doi.org/10.1590/S1415-52732003000400012.

Ministério da Saúde. (2019). Saúde Brasil 2018 uma análise da situação de saúde e das doenças e agravos crônicos: desafios e perspectivas. Ministério da Saúde, Secretaria de Vigilância em Saúde, Departamento de Vigilância de Doenças e Agravos Não Transmissíveis e Promoção da Saúde - Brasília: Ministério da Saúde, 424. http://bvsms.saude.gov.br/bvs/publicacoes/saude_brasil_2018_analise_situacao_saude_doencas_agravos_cronicos_desafios_perspectivas.pdf

Ministério da Saúde. (2012). Dor relacionada ao trabalho: lesões por esforços repetitivos (LER): distúrbios osteomusculares relacionados ao trabalho $(D O R T)$. Secretaria de Vigilância em Saúde Departamento de Vigilância em Saúde Ambiental e Saúde do Trabalhador. https://bvsms.saude.gov.br/bvs/publicacoes/dor_relacionada_trabalho_ler_dort.pdf 
Research, Society and Development, v. 10, n. 7, e44210716965, 2021

(CC BY 4.0) | ISSN 2525-3409 | DOI: http://dx.doi.org/10.33448/rsd-v10i7.16965

Ministério da saúde. (2001). Doenças relacionadas ao trabalho: Manual de procedimentos para os serviços de saúde. Brasília: Ministério da Saúde. https://www.paho.org/bra/index.php?option=com_docman\&view=download\&alias=207-doencas-relacionadas-ao-trabalho-manual-procedimentos-para-osservicos-saude-7\&category_slug=saude-e-ambiente-707\&Itemid=965

Oakman, J., Macdonald, W. \& Kinsman, N. (2019). Barriers to more effective prevention of work-related musculoskeletal and mental health disorders. Applied Ergonomics, 75, 184-192. https://doi.org/10.1016/j.apergo.2018.10.007

Oliveira, T. C. \& Silva, D. A. (2016). Administração de Unidades Produtoras de Refeições: desafios e perspectivas. Rubio.

Oliveira, V. C. \& Almeida, R. J. (2017). Aspects that determine the musculoskeletal disorders in nursing professionals and their psychosocial impact. Journal of Health Sciences, 19(2), 130-5. https://doi.org/10.17921/2447-8938.2017v19n2p130-135

Paes, I. C. E., Guilherme, R. C., Livera, A. V. de S., Valle, R. G. R. do \& Silveira, K. C. da (2020). Occupational risks of commercial restaurant workers in the metropolitan region of Recife-PE. Gestão \& Produção, 27(3), e4330. https://doi.org/10.1590/0104-530x4330-20

Park, S., Lee, J. \& Lee, J-H. (2021). Insufficient rest breaks at workplace and musculoskeletal disorders among korean kitchen workers. Safety and Health Work, 12(1), 1-5. https://doi.org/10.1016/j.shaw.2021.01.012

Pereira A. S., Shitsuka, D. M., Parreira, F. J., \& Shitsuka, R. (2018). Metodologia da pesquisa científica. UFSM. https://repositorio.ufsm.br/bitstream/handl e/1/158 24/Lic_Computacao_Metodologia-Pesquisa-Cientifica.pdf?sequence=1

Santos, I. N., Martins, I. C., Santos, A. C., Oliveira, A. S., \& Brasileiro-Santos, M. S. (2021). Work-related musculoskeletal disorders: a characterization of symptomatology and diagnostic exams. Research, Society and Development, 10(1), e36710111865. https://doi.org/10.33448/rsd-v10i1.11865

Sticca, M. G., Mandarini, M. B. \& Silva, F. L. M. Condições de trabalho e saúde de trabalhadores em um restaurante universitário. Estudos e Pesquisas em Psicologia, 19(3), 583-603. https://doi.org/10.12957/epp.2019.46904

Zavarizzi, C. P., Carvalho, R. M. M. \& Alencar, M. C. B. (2019). Grupos de trabalhadores acometidos por LER/DORT: relato de experiência. Cadernos Brasileiros de Terapia Ocupacional, 27(3), 663-670. https://doi.org/10.4322/2526-8910.ctore1756.

Wills, A., Davis, K. G. \& Kotowski, S. E. (2013). Quantification of the physical demands for servers in restaurants. Proceedings of the Human Factors and Ergonomics Society, 57(1), https://doi.org/10.1177/1541931213571219

World Health Organization - WHO (2000) Obesity: preventing and managing the global epidemic. WHO. https://apps.who.int/iris/handle/10665/42330 